\title{
Hand-Assisted Laparoscopic Donor Nephrectomy in Complete Situs Inversus
}

\author{
John V. Gahagan, MD, Matthew D. Whealon, MD, ${ }^{1}$ Uttam Reddy, MD, \\ Clarence E. Foster III, MD, and Hirohito Ichii MD, $\mathrm{PhD}^{1}$
}

\begin{abstract}
Complete situs inversus is a rare congenital anomaly characterized by transposition of organs. We report a case of renal transplantation using a kidney from a living complete situs inversus donor. The recipient was a 59-yearold female with end-stage renal disease because of type 2 diabetes mellitus. The donor was the 56-year-old sister of the recipient with complete situs inversus. CT angiogram of the abdomen and pelvis showed complete situs inversus and an otherwise normal appearance of the bilateral kidneys with patent bilateral single renal arteries and longer renal vein in the right kidney. The patient was taken to the operating room for a handassisted laparoscopic right donor nephrectomy. The patient tolerated the procedure well and was discharged home in good condition on postoperative day 1 . The recipient experienced no episodes of acute rejection or infection, with serum creatinine levels of $0.8-1.2 \mathrm{mg} / \mathrm{dL}$. Laparoscopic donor nephrectomy in a patient with complete situs inversus remains a technically feasible operation and the presence of situs inversus should not preclude consideration for living kidney donation.
\end{abstract}

\section{Introduction and Background}

$\mathbf{C}$ OMPLETE SITUS INVERSUS is a rare syndrome which has laterality reversal of thoracic and abdominal organs. It affects $\sim 1$ in 6000-8000 individuals and the etiology is not completely understood. ${ }^{1}$ The anatomic relationship between organs is unchanged, except that the configuration is a mirror image of the typical anatomic position. This reversal can give pause or confusion to physicians and surgeons performing an examination or a procedure. We present a contemporary case report of a hand-assisted laparoscopic donor nephrectomy in a patient with complete situs inversus.

\section{Presentation of Case}

The donor is a 56-year-old female with complete situs inversus who volunteered to donate a kidney to her sister. The recipient is a 59-year-old female with end-stage renal disease because of type 2 diabetes mellitus. The recipient has a history of hypertension for which she takes amlodipine. Her surgical history includes an open appendectomy and cesarean section. Laboratory values during preoperative planning were unremarkable, with a creatinine of $0.7 \mathrm{mg} / \mathrm{dL}$. Her BMI was 25.4 and her physical examination was otherwise normal in appearance. CT angiogram of the abdomen and pelvis showed situs inversus and an otherwise normal appearance of the bilateral kidneys with patent bilateral single renal arteries (Fig. 1 and 2). Echocardiography was unremarkable with a normal left ventricular ejection fraction. Mammography was normal.

The patient was taken to the operating room for a handassisted laparoscopic right donor nephrectomy. General anesthesia with orotracheal intubation was initiated and a Foley catheter was inserted. She was placed in the left lateral decubitus position with the bed flexed. A $7 \mathrm{~cm}$ vertical incision was made at the umbilicus through which we placed a wound protector and a GelPort (Applied Medical, Rancho Santa Margarita, CA). With a $5 \mathrm{~mm}$ trocar in the GelPort, the abdomen was insufflated to $15 \mathrm{mmHg}$ pressure. The patient was then given $12.5 \mathrm{~g}$ of mannitol. Two $5 \mathrm{~mm}$ trocars were placed: $3 \mathrm{~cm}$ (working) and $7 \mathrm{~cm}$ (camera) caudal from the right costal margin at the lateral border of the rectus abdominus. The left hand was inserted to the abdomen through the GelPort. After lysis of adhesions, the descending colon was mobilized along the white line of Toldt to the iliac vessels. The mesentery of the colon was dissected off the kidney, and Gerota's fascia was opened. We identified the gonadal vein and ureter, which were dissected together with fat and connective tissue. The ligamentous attachment between the

Departments of ${ }^{1}$ Surgery and ${ }^{2}$ Medicine, University of California, Irvine School of Medicine, Orange, California.

(C) John V. Gahagan et al. 2016; Published by Mary Ann Liebert, Inc. This Open Access article is distributed under the terms of the Creative Commons License (http://creativecommons.org/licenses/by/4.0), which permits unrestricted use, distribution, and reproduction in any medium, provided the original work is properly credited. 


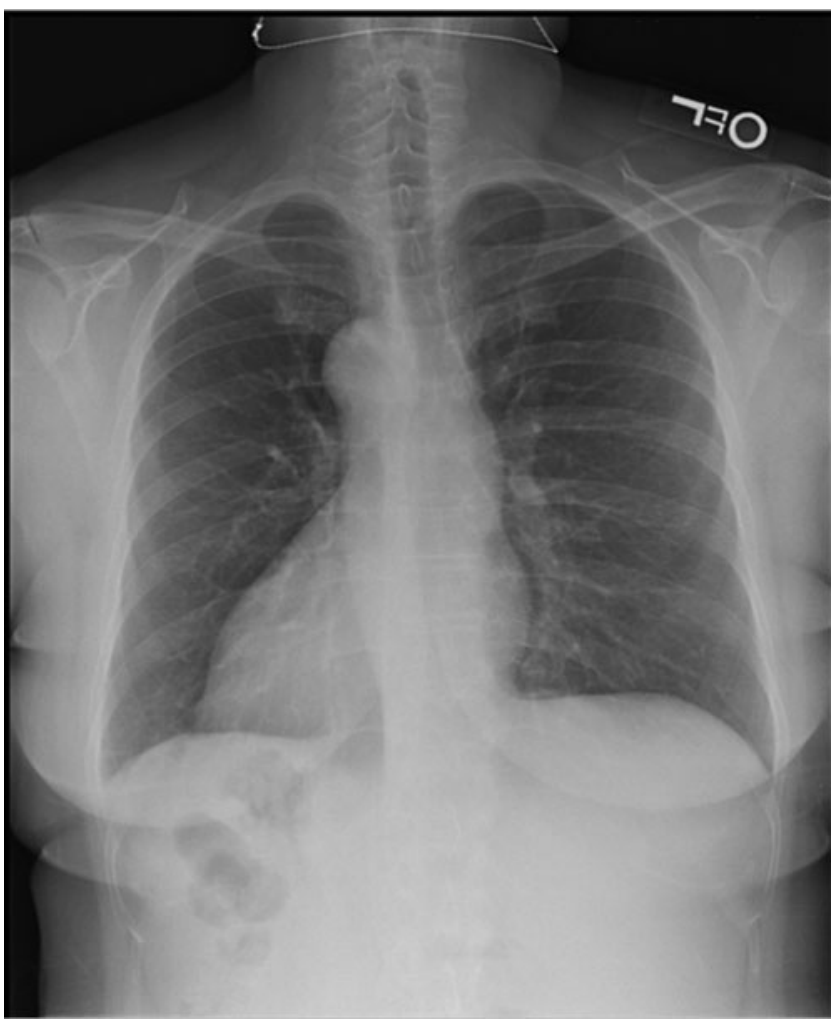

FIG. 1. Chest radiograph shows dextrocardia.

spleen and the upper pole of the kidney was identified and dissected. The right adrenal vein was identified at the medial side (Fig. 3). Since the length of right renal vein was enough for transplant, we elected to preserve the adrenal vein. The adrenal gland was identified and dissected from the kidney. The renal artery was located at the distal and posterior side of the right renal vein (Fig. 4). Once the kidney had been completely dissected free, and the renal artery and vein had been both isolated, heparin 5000 units and mannitol $12.5 \mathrm{~g}$ were given intravenously. The lower $5 \mathrm{~mm}$ trocar was upsized to a $12 \mathrm{~mm}$ trocar to accommodate a laparoscopic stapler. After 3 minutes, we divided the distal ureter and the

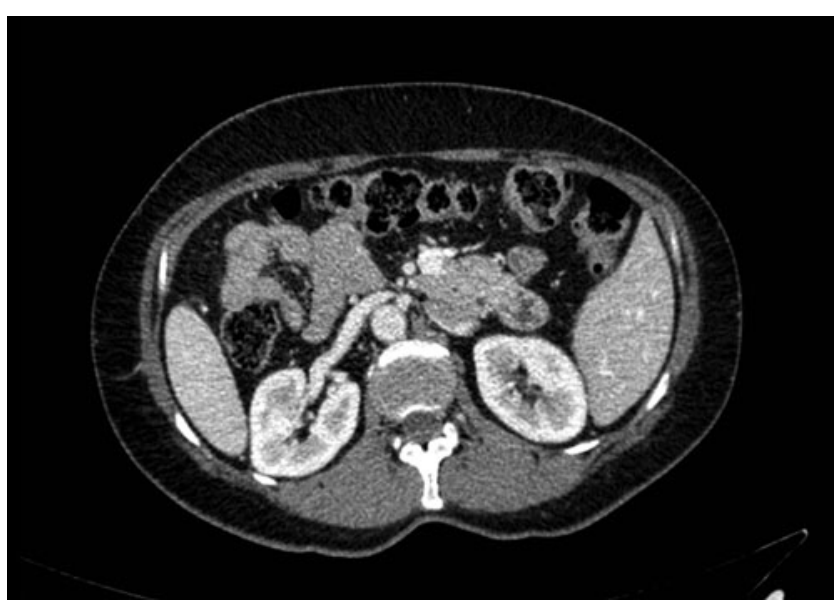

FIG. 2. Preoperative donor CT angiogram shows longer right renal vein.

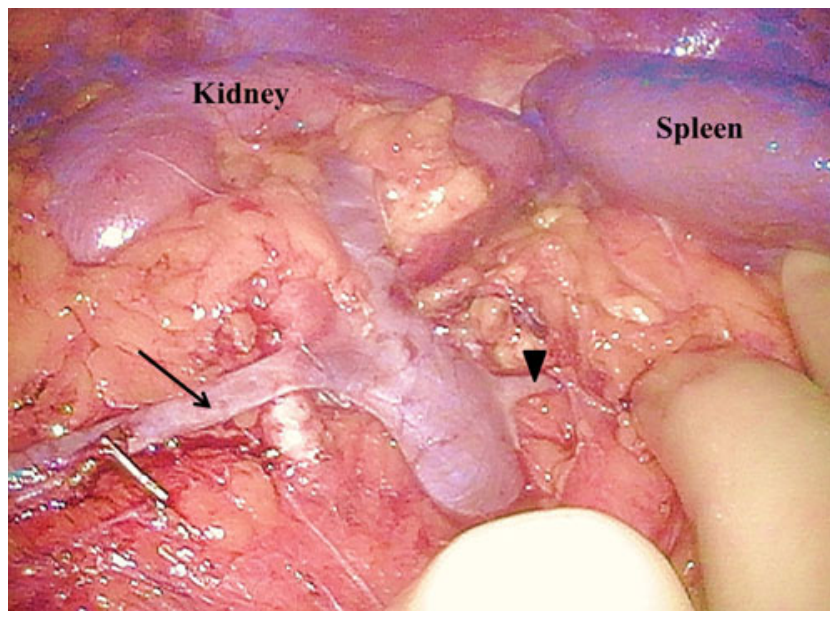

FIG. 3. The right gonadal vein (arrow) and right adrenal vein (arrowhead) of the donor were bifurcated from right renal vein.

gonadal vein with vascular stapler (ENDOPATH ${ }^{\circledR}$ ETS Articulating Linear Cutters, Ethicon). We then sequentially divided the renal artery and renal vein, each with the vascular stapler. The kidney was extracted through the midline incision. The warm ischemia time was less than 1 minute. The cold ischemia time was 85 minutes. The staple lines of both renal artery and vein were observed and were without evidence of bleeding. All incisions were closed. The patient tolerated the procedure well and was discharged home in good condition on postoperative day 1 .

\section{Discussion and Literature Review}

The technique of laparoscopic donor nephrectomy was first described in 1995 by Ratner et al. ${ }^{2}$ Since that time, the laparoscopic approach to a donor nephrectomy has been widely accepted and recognized as a safe procedure. ${ }^{3,4}$ In a majority of the laparoscopic donor nephrectomies, the left kidney is removed because of the technical ease of a longer vein and the absence of the liver and duodenum on that side. In the case of situs inverus, this anatomy is reversed, such that

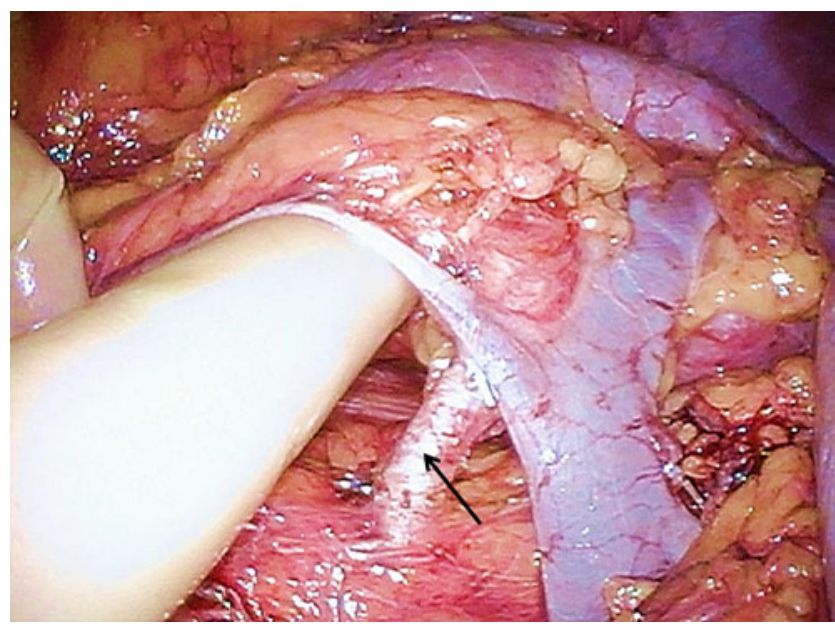

FIG. 4. Right renal artery (arrow) of the donor was identified behind renal vein. 
the patient's right kidney has a longer vein and the liver and duodenum are far away. In planning this case, we anticipated that we would place our incisions as though we were performing a standard right nephrectomy. After observing the right upper quadrant, this positioning seemed appropriate, although the anatomy was initially quite unique. The preoperative CT was crucial in our ability to anticipate the anatomy.

Laparoscopic appendectomy, cholecystectomy, and colectomy, among other procedures, in patients with compete situs inversus have previously been reported. The key conclusion in many of these reports is the importance of preoperative planning and understanding of the anatomy. This foresight allows surgeons to alter the operative technique before the operation and reorient their mind to the mirrored anatomy.

\section{Conclusion}

From our experience with this patient, laparoscopic donor nephrectomy in a patient with complete situs inversus remains a technically feasible operation and the presence of situs inversus should not preclude consideration for kidney donation.

\section{Acknowledgment}

There was no financial and material support.

\section{Disclosure Statement}

No competing financial interests exist.

\section{References}

1. Peeters H, Devriendt K. Human laterality disorders. Eur J Med Genet 2006;49:349-362.
2. Ratner LE, Ciseck LJ, Moore RG, Cigarroa FG, Kaufman HS, Kavoussi LR. Laparoscopic live donor nephrectomy. Transplantation 1995;60:1047-1049.

3. Jacobs SC, Cho E, Foster C, Liao P, Bartlett ST. Laparoscopic donor nephrectomy: The University of Maryland 6year experience. J Urol 2004;171:47-51.

4. Chin EH, Hazzan D, Herron DM, Gaetano JN, Ames SA, Bromberg JS, et al. Laparoscopic donor nephrectomy: Intraoperative safety, immediate morbidity, and delayed complications with 500 cases. Surg Endosc 2007;21:521-526.

Address correspondence to: Hirohito Ichii, MD, PhD, FACS

Division of Transplantation Department of Surgery University of California 333 City Boulevard West Suite 1205 Orange, CA 92868

E-mail: hichii@uci.edu

Cite this article as: Gahagan JV, Whealon MD, Reddy

U, Foster CE, III, Ichii H (2016) Hand-assisted laparoscopic donor nephrectomy in complete situs inversus, Journal of Endourology Case Reports 2:1, 108-110, DOI: 10.1089/cren.2016.0045. 